\title{
Associação de pós inertes com fungo entomopatogênico para o controle do cascudinho (Alphitobius diaperinus)
}

\author{
Association of inert powders with the entomopathogenic fungi for darkling beetle \\ (Alphitobius diaperinus) control
}

\author{
Patricia Helena Santoro ${ }^{I}$ Pedro Manuel Oliveira Janeiro Neves ${ }^{\text {II }}$ Junio Tavares Amaro ${ }^{\text {II }}$ \\ Kelly Constanski ${ }^{\text {II }}$ Ernesto Antonio Lema López ${ }^{\text {II }}$ Luis Francisco Angeli Alves ${ }^{\text {III }}$
}

\section{RESUMO}

O objetivo deste trabalho foi avaliar a eficiência de Beauveria bassiana (Bb), da terra diatomácea (TD) e dos pós de rocha Protesyl e Rocksil, isoladamente ou em associações, para o controle de Alphitobius diaperinus. Os agentes de controle foram aplicados na forma sólida misturados à cama de frango. A concentração de conídios de Bb foi de $0,08 \%$ e, para os pós inertes, as concentrações foram de 0,5; 1,0 e 1,5\%. Testaram-se 20 tratamentos, com cinco repetições de 200 insetos, por 10 dias. Os tratamentos que apresentaram maior eficiência foram TD 1,5\% e Bb 0,08\% + TD 1,5\%, com 60,2 e 57,8\% de mortalidade, respectivamente. Protesyl e Rocksil, nas três concentrações, e TD 0,5\% não diferiram da testemunha. Na interação entre os agentes de controle, ocorreu efeito aditivo para $B b+T D$ e $B b+$ Rocksil, nas três concentrações, e para Bb + Protesyl a 1,5\%. A eficiência do fungo foi reduzida na associação com Protesyl, com efeito não aditivo antagônico nas concentrações de 0,5 e 1,0\%. A terra diatomácea, isolada ou associada à B. bassiana, apresenta-se como boa alternativa para controle de A. diaperinus. Já os pós de rocha Protesyl e Rocksil não foram eficientes para o controle da praga.

Palavras-chave: aviários, Beauveria bassiana, controle biológico, controle microbiano.

\section{ABSTRACT}

The objective of this study was to evaluate the efficacy of Beauveria bassiana (Bb) of diatomaceous earth (TD) and the dust of rock Protesyl and Rocksil, alone or in combinations, for Alphitobius diaperinus to control. The control agents were applied in dried by mixing with the poultry liter. The concentration of Bb conidia was $0.08 \%$ on the weight of the substrate, and for the dust inert were 0.5, 1.0 and $1.5 \%$.
Twenty treatments with five repetitions of 200 insects were tested for 10 days. The treatments with highest efficiency were TD $1.5 \%$ and $\mathrm{Bb} 0.08 \%+T D 1.5 \%$, with 60.2 and $57.8 \%$, respectively. Protesyl and Rocksil in the three concentrations and TD $0.5 \%$ did not differ from control. For the interaction between control agents no advantage was observed for the association. Additive effect occurred for $B b+T D$ and $B b+$ Rocksil at the three concentrations and for Bb + Protesyl at $1.5 \%$. The action of the fungus was reduced in association with Protesyl with antagonistic non-additive effect in 0.5 and $1.0 \%$. Diatomaceous earth, isolated our in association with B. bassiana its a good alternative for A. diaperinus control. Just rock dust Protesyl and Rochsil were not efficient for insect control.

Key words: poultry houses, Beauveria bassiana, biological control, microbial control.

\section{INTRODUÇÃO}

A ocorrência de Alphitobius diaperinus (Panzer), em grandes populações nos aviários, fez desse inseto a principal praga no sistema de criação de aves. Essa espécie é originalmente citada como praga em grãos armazenados e foi introduzida nos aviários junto à ração destinada à alimentação das aves (O'CONNOR, 1987). Diversos trabalhos mostram que esse inseto pode ser reservatório e vetor de agentes patogênicos, como Coronavirus (WATSON et al., 2000); Proteus Vulgaris, Escherichia Coli, Enterobacter spp., Citrobacter diversus, Klebsiella Pneumoniae (CHERNAKI-LEFFER et al., 2002); Campylobacter spp.

Instituto Agronômico do Paraná (Iapar), Rodovia Celso Garcia Cid, km 375, 86047-902, Londrina, PR, Brasil. E-mail: ph_santoro@yahoo.com.br. Autor para correspondência.

"Universidade Estadual de Londrina (UEL), Londrina, PR, Brasil.

"I'Universidade do Oeste do Paraná (Unioeste), Cascavel, PR, Brasil 
(BATES et al., 2004); Salmonella enterica (SKOV et al., 2004); Klebsiella pneumoniae, Proteus mirabilis (SEGABINAZI et al., 2005) e Clostridium perfringens (VITTORI et al., 2007). O inseto também serve de alimento alternativo às aves, reduzindo o consumo de ração e consequentemente o ganho de peso (AXTELL \& ARENDS, 1990). A ingestão desses insetos pelas aves pode acarretar ainda contaminação da carcaça durante o processamento no abatedouro (CHERNAKILEFFER, 2004).

A permanência das aves nos aviários dificulta o controle de pragas com inseticidas químicos, que podem causar a intoxicação dos animais (ALVES et al., 2005). Além disso, a preocupação ambiental e a demanda por alimentos isentos de contaminantes fazem com que novas práticas na produção alimentar sejam adotadas em substituição aos modelos convencionais.

Entre os produtos permitidos para o controle de insetos em sistemas orgânicos estão os fungos entomopatogênicos, os pós inertes, como os pós de rocha, a terra diatomácea e alguns fitoterápicos, como os da família Meliaceae (BRASIL, 2008).

A ocorrência natural do fungo Beauveria bassiana (BALS.) em A. diaperinus sugere o potencial de controle desse patógeno, principalmente em razão das condições de temperatura e umidade da cama de frango, que podem favorecer epizootias (ALVES et al., 2005). Os fungos entomopatogênicos também apresentam a vantagem de serem inócuos aos animais endotérmicos (ALVES, 1998).

Os pós inertes, normalmente utilizados para controlar pragas em grãos armazenados, são substâncias provenientes de minerais extraídos de rochas que causam a morte de insetos por dessecação (LORINI, 1999). A terra diatomácea é um sedimento amorfo que se origina de organismos unicelulares, como algas microscópicas aquáticas (ANDRADE et al., 2001). É constituída predominantemente por dióxido de silício $(86 \%)$, que provoca danos à cutícula dos insetos pela adsorção da cera da epicutícula e pela abrasão da cutícula, o que a torna permeável à água e promove a morte do inseto por dessecação (KORUNIC, 1998). A atividade prolongada dos pós inertes, aliada à dificuldade dos insetos de desenvolverem resistência a eles, os fazem potencialmente competitivos para o controle de pragas. Além disso, tendem a ser mais uma opção de controle em regiões de clima seco, em razão da melhoria da eficiência de controle (ATUI et al., 2003).

Os pós de rocha são difundidos na agricultura orgânica como fornecedores de nutrientes para as plantas. Por serem ricos em silício, são associados também à resistência das plantas ao ataque de pragas e doenças (KORNDÖRFER \& DATNOFF,
1995). O seu efeito sobre o controle de insetos por contato, como acontece com a terra diatomácea, não tem sido estudado.

$\mathrm{Na}$ busca por alternativas para reduzir a população de $\boldsymbol{A}$. diaperinus com métodos que substituam o uso de inseticidas químicos, este trabalho teve como objetivo avaliar a eficiência do fungo $\boldsymbol{B}$. bassiana e de pós inertes, isoladamente e em associações, no controle da praga.

\section{MATERIAL E MÉTODOS}

$\mathrm{O}$ experimento foi realizado em condições de laboratório com adultos de $\boldsymbol{A}$. diaperinus coletados em aviário no Município de Londrina, Paraná (PR). Utilizou-se como substrato para os insetos a cama de frango onde viviam, constituída de maravalha e reutilizada pelo quinto lote de aves. A cama foi peneirada (malha de $2 \mathrm{~mm}$ ) para retirada dos insetos, fezes e outro material, e homogeneização do tamanho das partículas. Em caixas acrílicas com tampa (gerbox: $11 \times 11 \times 3,5 \mathrm{~cm}$ ), adicionaram-se $58 \mathrm{~g}$ de cama de frango e $2 \mathrm{~g}$ de ração de milho, utilizada para alimentar os insetos. O substrato ocupou cerca de $2 / 3$ do volume do recipiente.

Os agentes de controle testados de maneira isolada ou associados foram: conídios do isolado Unioeste 4 de B. bassiana (Bb), selecionado para o controle de $\boldsymbol{A}$. diaperinus (SANTORO et al., 2008b); terra diatomácea (TD) comercial Keepdry, composta por $86 \%$ de dióxido de sílica amorfa, com partículas de aproximadamente $15 \mu \mathrm{m}$; pó de rocha comercial Rocksil, composto de terra mineral, com $20,6 \% \mathrm{de}_{\mathrm{Al}} \mathrm{O}_{3} ; 17,4 \%$ de $\mathrm{SiO}_{2} ; 9,8 \%$ de $\mathrm{S} ; 1,3 \%$ de $\mathrm{CaO} ; 0,34 \%$ de $\mathrm{TiO}_{2} ; 0,18 \%$ de $\mathrm{MgO} ; 0,16 \%$ de $\mathrm{Fe}_{2} \mathrm{O}_{3}$ e $0,1 \%$ de $\mathrm{P}_{2} \mathrm{O}_{5}$; e o pó de rocha comercial Protesyl, constituído basicamente por argila mineral caulinita $\left(\mathrm{Al}_{2} \mathrm{Si}_{2} \mathrm{O}_{5}(\mathrm{OH})_{4}\right)$, com $46,5 \%$ de $\mathrm{SiO}_{2} ; 39,5 \% \mathrm{de}_{2} \mathrm{O}_{3}$ e $13,9 \% \mathrm{de}_{2} \mathrm{O}$ (GRIM, 1968).

As concentrações dos pós inertes foram de: 0,$5 ; 1,0$ e 1,5\%, em relação ao peso do substrato. Para o fungo, utilizou-se a concentração de $0,08 \%$ de conídios de B. bassiana. Os conídios foram produzidos em placas de Petri com meio BDA. Após a repicagem do fungo sobre o meio, as placas foram acondicionadas em câmara climatizada $\left(25 \pm 1^{\circ} \mathrm{C}\right.$ e fotófase de 12 horas $)$ por 10 dias, para o desenvolvimento do entomopatógeno. Em seguida, foram submetidos a teste prévio de germinação, segundo metodologia utilizada por SANTORO et al., 2008a, em que apresentaram viabilidade de $97 \%$. Sabendo-se que o isolado Unioeste 4 apresenta-se virulento à praga, o efeito de diferentes concentrações do fungo sobre os insetos foi testado previamente à realização, sendo responsável por aproximadamente $20 \%$ de mortalidade na concentração 
de $0,08 \%$. Optou-se por essa concentração, pois, quando se utiliza o método proposto por KOPPENHOFER et al. (2000), que avalia os efeitos das interações entre os agentes, a soma das mortalidades provocadas por cada agente deve ser inferior a $116 \%$ para que seja possível observar efeitos não aditivos sinérgicos ou antagônicos. Se a soma for igual ou superior a $116 \%$, os valores de $\chi^{2}$ calculado serão sempre inferiores ao tabelado, caracterizando interação aditiva.

Os agentes foram aplicados na forma sólida por meio de mistura manual ao substrato. No testemunho, os insetos foram mantidos no gerbox contendo apenas o substrato. O delineamento experimental foi inteiramente casualizado, com 20 tratamentos e cinco repetições com 200 insetos, que foram mantidos em câmara climatizada $\left(25 \pm 1^{\circ} \mathrm{C}\right.$ e fotófase de 12 horas). A avaliação foi realizada após 10 dias, pela contagem dos insetos mortos.

Os dados de mortalidade total foram submetidos à análise de variância, e as médias comparadas pelo teste de Tukey, a 5\% de probabilidade. Em seguida, foram corrigidos pela fórmula de Shneider e Orelli (NAKANO et al., 1981) e analisados pelo método proposto por KOPPENHOFER et al. (2000), que avalia a interação dos agentes de controle em associações pelo teste de qui-quadrado. Considera-se $\chi^{2}=(\mathrm{MFI}-$ $\mathrm{ME})^{2} / \mathrm{ME}$, em que MFI é a mortalidade no tratamento, sendo aplicados os agentes em associação; e ME é a mortalidade esperada, indicada como $\mathrm{ME}=\mathrm{MF}+\mathrm{MI} \times(1$ MF/100), MF é a mortalidade causada pelo fungo, e MI é a mortalidade provocada pelo pó inerte. Para $\chi^{2}$ significativo $(3,84$ para 1 grau de liberdade, $\mathrm{P}<0,05)$, o efeito foi considerado não aditivo sinérgico, quando MFI-ME $>0$, ou não aditivo antagônico, quando MFI$\mathrm{ME}<0$. Se $\chi^{2}$ foi não significativo, o efeito foi considerado aditivo.

\section{RESULTADOS E DISCUSSÃO}

Os maiores níveis de mortalidade total de $\boldsymbol{A}$. diaperinus foram observados em TD 1,5\%, com 60,2\%, e $\mathrm{Bb} 0,08 \%+$ TD $1,5 \%$, com $57,8 \%$ de controle. No tratamento contendo apenas B. bassiana, a mortalidade total foi de $18,3 \%$ (Tabela 1). A mortalidade total provocada pela ação da TD, para as concentrações de 0,5 a 1,5\%, variou de 4,1 a 60,2\%. Esses valores são inferiores aos constatados por ALVES et al., (2006), que utilizaram concentrações entre 0,1 e $0,3 \%$ de TD misturadas à ração e obtiveram mortalidade entre 70,0 e $90,0 \%$ ao décimo dia. A variação nos níveis de mortalidade, observada nos diferentes trabalhos, pode

Tabela 1 - Mortalidade total e corrigida de adultos de Alphitobius diaperinus (Panzer) ao $10^{\circ}$ dia após os tratamentos com pós inertes e Beauveria bassiana (Bals.), isolados e em associações.

\begin{tabular}{|c|c|c|}
\hline Tratamento $^{\mathrm{a}} \mathrm{n}=1.000$ & Mortalidade Total (\%) & Mortalidade Corrigida (\%) \\
\hline $\mathrm{Bb} 0,08 \%$ & $18,3 \pm 1,23 \mathrm{~cd}$ & 17,0 \\
\hline TD $0,5 \%$ & $4,1 \pm 0,87 \mathrm{hi}$ & 2,5 \\
\hline TD $1,0 \%$ & $20,6 \pm 1,61 \mathrm{c}$ & 19,3 \\
\hline TD $1,5 \%$ & $60,2 \pm 3,98 \mathrm{a}$ & 59,6 \\
\hline Protesyl 0,5\% & $4,2 \pm 0,44 \mathrm{hi}$ & 2,6 \\
\hline Protesyl 1,0\% & $4,9 \pm 1,36 \mathrm{ghi}$ & 3,4 \\
\hline Protesyl 1,5\% & $2,8 \pm 0,58 \mathrm{hi}$ & 1,2 \\
\hline Rocksil 0,5\% & $3,9 \pm 0,83 \mathrm{hi}$ & 2,3 \\
\hline Rocksil 1,0\% & $1,4 \pm 0,29 \mathrm{i}$ & $-0,2$ \\
\hline Rocksil 1,5\% & $3,6 \pm 0,66 \mathrm{i}$ & 2,0 \\
\hline $\mathrm{Bb} 0,08 \%+\mathrm{TD} 0,5 \%$ & $21,2 \pm 1,45 \mathrm{c}$ & 19,9 \\
\hline $\mathrm{Bb} 0,08 \%+\mathrm{TD} 1,0 \%$ & $31,0 \pm 2,69 b$ & 29,9 \\
\hline $\mathrm{Bb} 0,08 \%+\mathrm{TD} 1,5 \%$ & $57,8 \pm 1,60 \mathrm{a}$ & 57,1 \\
\hline Bb $0,08 \%+$ Protesyl $0,5 \%$ & $9,8 \pm 0,77 \mathrm{fgh}$ & 8,3 \\
\hline Bb $0,08 \%+$ Protesyl $1,0 \%$ & $12,4 \pm 1,04 \mathrm{def}$ & 11,0 \\
\hline Bb $0,08 \%+$ Protesyl $1,5 \%$ & $14,4 \pm 1,29$ cde & 13,0 \\
\hline $\mathrm{Bb}$ 0,08\% + Rocksil 0,5\% & $19,5 \pm 1,26 \mathrm{~cd}$ & 18,2 \\
\hline Bb $0,08 \%+$ Rocksil $1,0 \%$ & $21,7 \pm 1,54 \mathrm{c}$ & 20,4 \\
\hline $\mathrm{Bb} 0,08 \%+$ Rocksil 1,5\% & $18,5 \pm 1,13 \mathrm{~cd}$ & 17,2 \\
\hline Testemunha & $1,6 \pm 0,29 \mathrm{i}$ & 0,0 \\
\hline
\end{tabular}

${ }^{\mathrm{a}} \mathrm{TD}=$ terra diatomácea, $\mathrm{Bb}=$ Beauveria bassiana $;{ }^{\mathrm{b}}$ Médias ( \pm erro-padrão) seguidas de mesma letra não diferem entre si, a $5 \%$ de probabilidade; CV: 20,19\%. 
ser explicada pelos substratos utilizados, os quais interferem na ação da TD no controle de insetos. ALVES et al. (2008) verificaram que a TD foi responsável por 4,0\% da mortalidade de $A$. diaperinus, quando os insetos foram acondicionados em cama de frango, e 95,5\%, quando acondicionados em ração.

Os pós de rocha Protesyl e Rocksil, nas três concentrações, não apresentaram ação sobre $\boldsymbol{A}$. diaperinus, com valores de mortalidades entre 1,4 e $4,9 \%$ (Tabela 1). Esse resultado pode ser explicado pela menor concentração de dióxido de silício nos pós de rocha (17,4\% no Rocksil e $46,5 \%$ no Protesyl) em comparação à concentração existente na TD $(86,0 \%)$, visto que é o $\mathrm{SiO}_{2}$ o responsável pelos danos à cutícula dos insetos e pela promoção da morte por dessecação (KORUNIC, 1998). Para TD 0,5\%, a mortalidade também não diferiu do testemunho.

Ao analisar a interação dos agentes de controle, verificou-se efeito aditivo para a associação $\mathrm{de} \mathrm{Bb}+\mathrm{TD}$ e $\mathrm{Bb}+$ Rocksil, nas três concentrações, e para a associação de Bb + Protesyl, a 1,5\% (Tabela 2). AKBAR et al. (2004) também não observaram incremento na mortalidade de Tribolium castaneum (HERBST), na associação de B. bassiana com TD. Já para Rhyzopertha dominica (Fabricius) e Tribolium confusum (Jacquelin Du Val) essa associação favoreceu o controle em grãos armazenados (VAYAS \& ATHANASSIOU, 2004; LORD, 2005). Efeito não aditivo sinérgico foi observado no controle de $A$. diaperinus, na associação de $\boldsymbol{B}$. bassiana e TD, em experimento realizado por SANTORO et al. (2008a).
A variação dos efeitos nos diferentes trabalhos em que a TD foi associada à $\boldsymbol{B}$. bassiana pode estar relacionada com variações nos teores de umidade dos substratos ou dos ambientes, que exercem influência tanto na ação do fungo, como na ação da TD, porém esses fatores não foram avaliados nesses experimentos. Segundo KORUNIC (1998), a eficiência da TD está relacionada principalmente à baixa umidade do ambiente tratado. As partículas de TD, ao absorverem água, tornam-se menos eficazes (ARTHUR, 2001), assim esse pó é mais eficiente em condições de clima seco (ATUI et al., 2003). Isso foi constatado para o controle de $A$. diaperinus, em que a eficiência da TD, do fungo $\boldsymbol{B}$. bassiana e da associação de ambos foi maior em condições de baixa umidade relativa (OLIVEIRA\&ALVES, 2007).

LORD (2005) verificou que a eficiência de TD e B. bassiana, para o controle de $R$. dominica, foi maior em condições de baixa umidade. Um aumento de $55 \%$ para $65 \%$ na umidade relativa reduziu a mortalidade de T. confusum, por TD, em aproximadamente $10 \%$ (VAYAS \& ATHANASSIOU, 2004). Entretanto, quando a umidade do substrato não pode ser controlada, como acontece nos aviários, recomenda-se o aumento da concentração de TD para manutenção da eficiência de controle (KORUNIC, 1998).

Os efeitos aditivos observados neste estudo e os níveis de mortalidade provocados pela TD e $\boldsymbol{B}$. bassiana mostram que esses agentes apresentam potencial de controle e podem ser incluídos no manejo de A. diaperinus, juntamente com outros métodos de

Tabela 2 - Interação do fungo Beauveria bassiana (Bals.) associado aos pós inertes como agentes controladores de adultos de Alphitobius diaperinus (Panzer) ao $10^{\circ}$ dia após os tratamentos.

\begin{tabular}{|c|c|c|c|c|}
\hline \multirow{2}{*}{ Tratamento $^{\mathrm{a}} \mathrm{n}=1000$} & \multicolumn{2}{|c|}{ Mortalidade (\%) } & \multirow{2}{*}{$\chi^{2 \mathrm{~d}}$} & \multirow{2}{*}{ Interação } \\
\hline & Observada $^{b}$ & Esperada $^{c}$ & & \\
\hline $\mathrm{Bb} 0,08 \%+\mathrm{TD} 0,5 \%$ & 19,9 & 19,1 & 0,04 & Aditiva \\
\hline $\mathrm{Bb} 0,08 \%+\mathrm{TD} 1,0 \%$ & 29,9 & 33,0 & 0,30 & Aditiva \\
\hline $\mathrm{Bb} 0,08 \%+\mathrm{TD} 1,5 \%$ & 57,1 & 66,4 & 1,30 & Aditiva \\
\hline $\mathrm{Bb} 0,08 \%+$ Protesyl $0,5 \%$ & 8,3 & 19,2 & 6,12 & Não-aditiva antagônica \\
\hline $\mathrm{Bb} 0,08 \%+$ Protesyl $1,0 \%$ & 11,0 & 19,8 & 3,90 & Não-aditiva antagônica \\
\hline $\mathrm{Bb} 0,08 \%+$ Protesyl $1,5 \%$ & 13,0 & 18,0 & 1,38 & Aditiva \\
\hline $\mathrm{Bb} 0,08 \%+$ Rocksil 0,5\% & 18,2 & 18,9 & 0,03 & Aditiva \\
\hline $\mathrm{Bb} 0,08 \%+$ Rocksil 1,0\% & 20,4 & 16,8 & 0,78 & Aditiva \\
\hline $\mathrm{Bb} 0,08 \%+$ Rocksil $1,5 \%$ & 17,2 & 18,7 & 0,12 & Aditiva \\
\hline
\end{tabular}

${ }^{\mathrm{a}} \mathrm{TD}=$ terra diatomácea, $\mathrm{Bb}=\boldsymbol{B}$. bassiana $;{ }^{\mathrm{b}} \mathrm{Mortalidade}$ observada, corrigida pela forma de Schneider e Orelli; ${ }^{\mathrm{c}} \mathrm{Mortalidade}$ esperada (ME), calculada pela fórmula: $\mathrm{ME}=\mathrm{MF}+\mathrm{MI} *(1-\mathrm{MF} / 100)$, em que MF é a mortalidade causada pelo fungo, e MI é a mortalidade provocada pelo pó inerte; $\left.{ }^{\mathrm{d}} \chi^{2}=(\mathrm{MFI}-\mathrm{ME})^{2}\right) / \mathrm{ME}$, em que MFI é a mortalidade na associação do fungo com o pó inerte; $\chi^{2}$ (tabelado)=3,84; Grau de liberdade = 1 ; $\mathrm{P}>0,05$. 
controle. Os efeitos aditivos em que TD e B. bassiana foram associados podem estar relacionados à compatibilidade entre os agentes, pois estudos mostram que TD é inócua à B. bassiana (OLIVEIRA \& ALVES, 2007).

Em relação aos pós de rochas testados, não existem relatos sobre a compatibilidade com fungos entomopatogênicos, porém observou-se neste estudo um efeito não aditivo antagônico na associação de $\mathrm{Bb}$ + Protesyl, nas concentrações de 0,5 e 1,0\% (Tabela 2). $\mathrm{O}$ efeito antagônico provocado pelo Protesyl sobre o fungo $\boldsymbol{B}$. bassiana pode ter ocorrido pelo elevado teor de óxido de alumínio $\mathrm{Al}_{2} \mathrm{O}_{3}$ presente na caulinita, que, de acordo com GRIM (1968), é de 39,5\%. O alumínio é um elemento utilizado na composição de alguns fungicidas na agricultura. Apesar do efeito negativo sobre o fungo entomopatogênico no controle de $\boldsymbol{A}$. diaperinus, esses resultados podem incentivar estudos que tenham como objetivo testar a utilização do Protesyl no controle de doenças fúngicas em plantas. No tratamento $\mathrm{Bb}+$ Protesyl 1,5\%, o efeito aditivo pode estar relacionado ao pequeno aumento na mortalidade $(2,0 \%)$, que, apesar de não diferir significativamente do tratamento $\mathrm{Bb}+$ Protesyl $1,0 \%$, foi suficiente para não ser evidenciado o efeito antagônico.

\section{CONCLUSÕES}

A terra diatomácea apresenta potencial de controle para A. diaperinus quando aplicada na forma de pó sobre a cama de frango, e sua ação não é antagônica ao fungo $\boldsymbol{B}$. bassiana. Isso significa que esses agentes de controle podem ser utilizados no manejo das populações de cascudinho com aplicação em associação, mesmo não apresentando efeito sinérgico. Já os pós de rocha Rocksil e Protesyl não são eficientes para o controle de $\boldsymbol{A}$. diaperinus.

\section{AGRADECIMENTOS}

Ao produtor Anízio Fecchio, proprietário do aviário onde foram feitas as coletas dos insetos, e ao professor Dr. Ayres Menezes, do Departamento de Agronomia da Universidade Estadual de Londrina, por fornecer os pós de rocha utilizados neste estudo.

\section{REFERÊNCIAS}

AKBAR, W. et al. Diatomaceous earth increases the efficacy of Beauveria bassiana against Tribolium castaneum larvae and increases conidia attachment. Journal of Economic Entomology, v.97, p.273-280, 2004. Disponível em: <http://bru.gmprc.ksu.edu/ pdf/861_EcEn_97.273.pdf $>$. Acesso em: 06 maio, 2010. doi: 10.1603/0022-0493(2005)098[0683:EOBBFR]2.0.CO;2.
ALVES, L.F.A. et al. Ação da terra de diatomácea contra adultos do cascudinho Alphitobius diaperinus (Panzer, 1797) (Coleoptera: Tenebrionidae). Arquivos do Instituto Biológico, v.73, p.115118, 2006. Disponível em: <http://www.biologico.sp.gov.br/docs/ arq/V73_1/alves.PDF>. Acesso em: 26 jan. 2009.

ALVES, L.F.A. et al. Fatores a serem considerados na utilização de Beauveria bassiana visando o manejo de populações de Alphitobius diaperinus em aviários comerciais. Arquivos do Instituto Biológico, v.75, p.13-20, 2008. Disponível em: $<$ http://www.biologico.sp.gov.br/docs/arq/v75_1/alves.pdf.>. Acesso em: 01 mar. 2009.

ALVES, L.F.A. et al. Ocorrência natural de Beauveria bassiana (Bals.) Vuilleman (Moniliales: Moniliaceae) sobre o cascudinho (Alphitobius diaperinus) (Panzer) (Coleoptera: Tenebrionidae), em aviários comerciais de Cascavel, PR. Neotropical Entomology, v.34, p.507-510, 2005. Disponível em: <http:/ $/$ www.scielo.br/scielo.php?script $=$ sci_arttext\&pid $=\mathrm{S} 1519$ 566X2005000300021\&lng=pt\&nrm=iso $>$. Acesso em: 01 mar 2009. doi: 10.1590/S1519-566X2005000300021.

ALVES, S.B. Fungos entomopatogênicos. In: ALVES, S.B. (Ed.). Controle microbiano de insetos. Piracicaba: FEALQ, 1998. p.289-381.

ANDRADE, M.S. et al. Especiação de sílica e estudo de metodologias de digestão de diatomito assistida por microondas com radiação focalizada. In: CONGRESSO ANUAL DA ABM INTERNACIONAL, 56., 2001, Belo Horizonte. Anais... Belo Horizonte: ABM Internacional, 2001. p.1019-1028.

ARTHUR, F.H. Immediate and delayed mortality of Oryzaephilus surinamensis (L.) exposed on wheat treated with diatomaceous earth: effects of temperature, relative humidity, and exposure interval. Journal of Stored Products Research, v.37, p.646649, 2001. Disponível em: <http://ddr.nal.usda.gov/bitstream/ 10113/12580/1/IND22435525.pdf >. Acesso em: 06 maio, 2010. doi: 10.1016/S0022-474X(99)00058-2.

ATUI, M.B. et al. Evaluation of the methodology for detection of diatomaceous earth residue in wheat grain and flour. Revista do Instituto Adolfo Lutz, v.62, p.11-16, 2003. Disponível em: <http://biblioteca.ial.sp.gov.br/ index.php?option $=$ com_remository\&Itemid $=27 \&$ func $=$ startdown\&id $=281>$. Acesso em: 23 jan. 2009.

AXTELL, R.C.; ARENDS, J.J. Ecology and management of arthropod pests of poultry. Annual Review of Entomology, v.35, p.101-126, 1990. Disponível em: <http:// repository.lib.ncsu.edu/publications/bitstream/1840.2/2120/1/ axtell+1990.pdf $>$. Acesso em: 06 maio, 2010. doi: 10.1146/ annurev.en.35.010190.000533.

BATES, C. et al. Relationship of Campylobacter isolated from poultry and from darkling beetles in New Zealand. Avian Diseases, v.48, p.138-147, 2004. doi: 10.1637/7082.

BRASIL. Instrução Normativa n. 64, de 18 de dezembro de 2008. Aprova o Regulamento Técnico para os Sistemas Orgânicos de Produção Animal e Vegetal. Diário Oficial da União, Brasília, 18 dez. 2008. Seção 1, 21p. Disponível em: $<\mathrm{http}$ ://extranet.agricultura.gov.br/sislegis-consulta/ consultarLegislacao. $d o$ ?operacao $=$ visualizar\&id $=19345>$. Acesso em: 08 mar. 2009. 
CHERNAKI-LEFFER, A.M. et al. Isolamento de enterobactérias em Alphitobius diaperinus e na cama de aviários no oeste do Paraná, Brasil. Revista Brasileira de Ciência Avícola, v.4, p.243-247, 2002. Disponível em: <http:// www.scielo.br/scielo.php?script $=$ sci_arttext\&pid $=$ S 1516 $635 X 2002000300009 \& \operatorname{lng}=$ pt\&nrm=iso $>$. Acesso em: 12 abr. 2009. doi: $10.1590 / \mathrm{S} 1516-635 \times 2002000300009$.

CHERNAKI-LEFFER, A.M. Dinâmica populacional, estimativa da resistência a inseticidas e alternativas de controle para o cascudinho Alphitobius diaperinus (Panzer, 1797) (Coleoptera: Tenebrionidae). 2004. 123f. Tese (Doutorado em Ciências) - Universidade Federal do Paraná, Curitiba, PR.

GRIM, R.E. Clay mineralogy. New York: McGraw-Hill, 1968. 596p.

KOPPENHOFER, A.M. et al. Synergism of entomopathogenic nematodes and imidacloprid against white grubs: greenhouse and field evaluation. Biological Control, v.19, p.245-251, 2000

KORNDÖRFER, G.H.; DATNOFF, L.E. Adubação com silício: uma alternativa no controle de doenças da cana-de-açúcar e do arroz. Informações Agronômicas, v.70, p.1-3, 1995.

KORUNIC, Z. Diatomaceous earths, a group of natural insecticides. Journal of Stored Products Research, v.4, p.87-97, 1998.

LORD, J.C. Low humidity, moderate temperature, and desiccant dust favor efficacy of Beauveria bassiana (Hyphomycetes: Moniliales) for the lesser grain borer, Rhyzopertha dominica (Coleoptera: Bruchidae). Biological Control, v.34, p.180$186,2005$.

LORINI, I. Pragas de grãos de cereais armazenados. Passo Fundo: Embrapa Trigo, 1999. 60p.

NAKANO, O. et al. Entomologia econômica. São Paulo: Ceres, 1981. 314p.

O'CONNOR, J.P. Alphitobius diaperinus (Panzer) (Col.: Tenebrionidae) damaging polystyrene insulation in Irish piggery. Entomologist's Monthly Magazine, v.123, p.1472-1475, 1987.

OLIVEIRA, D.G.P.; ALVES, L.F.A. Interação do fungo Beauveria bassiana (Bals.) Vuill. com terra diatomácea para o controle de Alphitobius diaperinus (Panzer) (Coleoptera: Tenebrionidae), o cascudinho dos aviários. BioAssay, v.2, p.6, 2007. Disponível em: <http://www.bioassay.org.br/articles/2.6/ BA2.6.pdf>. Acesso em: 24 abr. 2009.

SANTORO, P.H. et al. Controle associado de Alphitobius diaperinus e efeito de microrganismos eficazes no desenvolvimento de Beauveria bassiana. Pesquisa Agropecuaria Brasileira, v.43, p.1-8, 2008a. Disponínel em: $<$ http://www.scielo.br/scielo.php?script=sci_arttext\&pid=S0100204X2008000100001\&lng=pt\&nrm=iso >. Acesso em: 21 jan. 2009. doi: 10.1590/S0100-204X2008000100001.

SANTORO, P.H. et al. Selection of Beauveria bassiana isolates to control Alphitobius diaperinus. Journal of Invertebrate Pathology, v.97, p.83-90, 2008b.

SEGABINAZI, S.D. et al. Bactérias da família Enterobacteriaceae em Alphitobius diaperinus oriundos de granjas avícolas dos Estados do Rio Grande do Sul e Santa Catarina, Brasil. Acta Scientiae Veterinariae, v.33, p.51-55, 2005. Disponível em: <http:// www.ufrgs.br/actavet/33-1/artigo608.pdf $>$. Acesso em: 21 jan. 2009.

SKOV, M.N. et al. The role of litter beetles as potential reservoir for Salmonella enterica spp and Thermophilic Campylobacter spp. between broiler flocks. Avian Diseases, v.48, p.9-18, 2004.

VAYIAS, B.J.; ATHANASSIOU, C.G. Factors affecting the insecticidal efficacy of the diatomaceous earth formulation SilicoSec against adults and larvae of the confused flour beetle, Tribolium confusum DuVal (Coleoptera: Tenebrionidae). Crop Protection, v.23, p.565-573, 2004.

VITTORI, J. et al. Alphitobius diaperinus como veiculador de Clostridium perfingens em granjas avícolas do interior paulista - Brasil. Ciência Rural, v.37, p.894-896, 2007. Disponível em: <http://www.scielo.br/pdf/cr/v37n3/a48v37n3.pdf $>$. Acesso em: 11 nov. 2008.

WATSON, D.W.et al. Limited transmission of Turkey Coronavirus in Yong Turkeys by adult Alphitobius diaperinus (Coleoptera: Tenebrionidae). Journal of Medical Entomology, v.37, p.480-483, 2000. Disponível em: <http:/ /www.entsoc.org/pubs/Periodicals/jme/JMETOCS/PDF/ me030000480p.pdf $>$. Acesso em: 12 mar. 2009. doi: 10.1603/ 0022-2585(2000)037[0480:LTOTCI]2.0.CO;2. 